\section{Eccentric Exercise in COPD}

\section{Take It or Leave It?}

\author{
Joana Cruz, PT, PhD \\ Leiria, Portugal \\ Chris Burtin, PT, PhD \\ Hasselt, Belgium
}

Eccentric training has been proposed as a therapeutic alternative to concentric training in symptom-limited individuals with COPD who are unable to tolerate conventional exercise, because up to four times greater muscle forces can be generated with lower ventilatory demands. ${ }^{1}$ Scientific evidence on eccentric training in COPD has emerged in the past decade,$^{2-7}$ and studies have shown that endurance training with the use of eccentric muscle contractions is well-tolerated, safe and feasible. ${ }^{7}$ However, evidence on its ability to elicit sufficient physiologic responses to induce a training effect similar to concentric training is still under debate, and it is unclear whether responses are comparable between patients with COPD and healthy individuals.

In this issue of CHEST, Ward et $\mathrm{al}^{8}$ compare the acute whole-body cardiopulmonary responses between eccentric and concentric cycling tests across a range of matched mechanical workloads in stable patients with COPD and age-matched healthy control subjects. Participants cycled for up to six 4-minute bouts of concentric and eccentric exercise, starting at $15 \%$ of the peak workload achieved in a maximal concentric incremental cardiopulmonary test, with $15 \%$ increments

FOR RELATED ARTICLE, SEE PAGE 564

AFFILIATIONS: From the Center for Innovative Care and Health Technology (ciTechcare), School of Health Sciences (ESSLei), Polytechnic of Leiria (J. Cruz); and Rehabilitation Research Center (REVAL), Biomedical Research Institute (BIOMED), Faculty of Rehabilitation Sciences, Hasselt University (C. Burtin).

FINANCIAL/NONFINANCIAL DISCLOSURES: None declared.

CORRESPONDENCE TO: Joana Cruz, PT, PhD; e-mail: joana.cruz@ ipleiria.pt

Copyright (c) 2020 American College of Chest Physicians. Published by Elsevier Inc. All rights reserved.

DOI: https://doi.org/10.1016/j.chest.2020.10.021 until reaching $90 \%$ of the peak workload or volitional exhaustion. Differences between eccentric and concentric cycling were comparable in patients and healthy control subjects. For the same workload, the magnitude of acute cardiopulmonary responses was lower in eccentric than concentric cycling, with minimal increases in oxygen uptake and minute ventilation even at higher workloads, and a lower perception of fatigue and dyspnea. In separate visits, the authors also performed muscle biopsies to determine muscle metabolic responses in patients with COPD before and after 20-minute (or until volitional exhaustion) eccentric and concentric cycling at a constant matched mechanical load of $65 \%$ of the peak workload. Changes in phosphocreatine, creatine, and lactate concentrations were observed after concentric, but not eccentric, cycling. Findings suggest that, at the same mechanical loads, eccentric cycling does not elicit similar acute cardiopulmonary and metabolic responses as concentric cycling and evokes lower exercise-induced symptoms. It should be noted that patients are expected to be able to achieve higher absolute mechanical loads during eccentric cycling.

Two recent randomized controlled trials evaluated the chronic effects (adaptations) of eccentric vs concentric cycling in patients with advanced COPD. ${ }^{5,6}$ Both studies were conducted by the same research group and included 10 weeks of training that consisted of three weekly 30-minute sessions of eccentric or concentric cycling. These studies found that eccentric cycling elicited a greater improvement of peak isometric quadriceps strength and relative thigh mass and showed less muscle mitochondrial adaptation compared with concentric cycling. The latter is in line with the low metabolic responses at the muscle level observed in Ward et al. ${ }^{8}$

Interestingly, literature suggests that eccentric training (both eccentric cycling and downhill walking) can enhance whole-body exercise capacity, possibly to a similar extent as concentric training. ${ }^{3,5}$ This matches with the observed strong relationship between muscle strength and exercise capacity in COPD. Lower limb muscle weakness is common in COPD and related to exercise intolerance, impaired postural control, poor quality of life, and premature death. ${ }^{9}$ Therefore, 
optimizing muscle strength is considered an important goal of rehabilitation. It is tempting to speculate that eccentric cycling could particularly be an interesting intervention in patients with profound leg muscle weakness who are markedly limited by dyspnea during moderate-to-high intensity aerobic exercise.

The equipment used may play a role in the physiologic response and subjective experience of eccentric exercise. Ward et $\mathrm{al}^{8}$ used an upright cycle ergometer to perform the tests, which may limit trunk stability and reaction forces required for eccentric force production. ${ }^{10}$ This was supported by participants who reported that eccentric cycling was challenging due to the concentration required and the difficulty in getting used to the technique. ${ }^{8}$ Recumbent cycle ergometers may be an alternative because patients do not demonstrate motor coordination difficulties during eccentric cycling, except at higher work rates $(>176 \mathrm{~W})$, although it does not seem to prevent the progression of power in subsequent training sessions. ${ }^{7}$ Another alternative is the use of downhill walking, which can be easier to implement and less expensive than cycle ergometers with an eccentric function. In COPD, downhill walking (eccentric exercise) produced lower acute cardiopulmonary effects than level walking (concentric exercise), similarly to the findings of Ward et $\mathrm{al}^{8}$, while inducing greater low-frequency fatigue in the quadriceps muscle (a determinant of optimal response to exercise in terms of exercise capacity). ${ }^{2}$ Walking training has the advantage of being translated easily to a daily functional activity and involving several muscles from the lower limb, while cycling places greater stress in the quadriceps muscle. The choice of the equipment should consider patients' tolerance levels, available resources, and training goals.

Overall, eccentric training is a safe and alternative exercise modality for patients with stable COPD to improve muscle function at reduced ventilatory costs. However, evidence of its effects on aerobic capacity is still scarce. The small samples and the heterogeneity of COPD grades from previous studies preclude firm conclusions about which patients may benefit the most from this type of training. It is widely recognized that patients' response to exercise is highly variable and compliance with exercise programs may be compromised in severely symptom-limited patients, thus tailored interventions are recommended to optimize outcomes. ${ }^{11}$ Eccentric training may be a feasible option for these patients in the early stages of rehabilitation to motivate them to adhere to exercise while improving muscle function. Ward et $\mathrm{al}^{8}$ found that most patients preferred eccentric to concentric cycling, which supports this idea. Further research is needed to standardize the intensity, duration, and modes of eccentric training and to determine the best candidates and timing within pulmonary rehabilitation to optimize its application and ensure patient safety.

\section{References}

1. Ellis R, Shields N, Lim K, Dodd KJ. Eccentric exercise in adults with cardiorespiratory disease: a systematic review. Clin Rehabil. 2015;29(12):1178-1197.

2. Camillo CA, Burtin C, Hornikx M, et al. Physiological responses during downhill walking: a new exercise modality for subjects with chronic obstructive pulmonary disease? Chron Respir Dis. 2015;12(2):155-164.

3. Camillo CA, Osadnik CR, Burtin C, et al. Effects of downhill walking in pulmonary rehabilitation for patients with COPD: a randomised controlled trial. Eur Respir J. 2020;56(3):2000639.

4. Mujaddadi A, Moiz JA, Singla D, Naqvi IH, Ali MS, Talwar D. Effect of eccentric exercise on markers of muscle damage in patients with chronic obstructive pulmonary disease [published online ahead of print July 25, 2019]. Physiother Theory Pract. 2019:1-7. https://doi. org/10.1080/09593985.2019.1644690.

5. Bourbeau J, De Sousa Sena R, Taivassalo T, et al. Eccentric versus conventional cycle training to improve muscle strength in advanced COPD: a randomized clinical trial. Respir Physiol Neurobiol. 2020;276:103414.

6. MacMillan NJ, Kapchinsky S, Konokhova Y, et al. Eccentric ergometer training promotes locomotor muscle strength but not mitochondrial adaptation in patients with severe chronic obstructive pulmonary disease. Front Physiol. 2017;8(114):1-14.

7. Rocha Vieira DS, Baril J, Richard R, Perrault H, Bourbeau J, Taivassalo T. Eccentric cycle exercise in severe COPD: feasibility of application. COPD. 2011;8(4):270-274.

8. Ward TJC, Lindley MR, Ferguson RA, et al. Submaximal eccentric cycling in people with COPD: acute whole-body cardiopulmonary and muscle metabolic responses. Chest. 2021;159(2):564-574.

9. Spruit MA, Singh SJ, Garvey C, et al. An official American Thoracic Society/European Respiratory Society statement: key concepts and advances in pulmonary rehabilitation. Am J Respir Crit Care Med. 2013;188(8):e13-e64.

10. Lipski M, Abbiss CR, Nosaka K. Cardio-pulmonary responses to incremental eccentric and concentric cycling tests to task failure. Eur J Appl Physiol. 2018;118(5):947-957.

11. Spruit MA, Wouters EFM. Organizational aspects of pulmonary rehabilitation in chronic respiratory diseases. Respirology. 2019;24(9):838-843. 